\title{
Investigation of the Photocatalytic Activity of ZnO Nanowires: Substrate Effect and Kinetics Analysis
}

\author{
Jan-Hau Chang and Heh-Nan Lin \\ Department of Materials Science and Engineering, National Tsing Hua University, Hsinchu 30013, Taiwan \\ Correspondence should be addressed to Heh-Nan Lin; hnlin@mx.nthu.edu.tw
}

Received 24 January 2014; Accepted 1 June 2014; Published 1 July 2014

Academic Editor: Yanbao Zhao

Copyright ( 2014 J.-H. Chang and H.-N. Lin. This is an open access article distributed under the Creative Commons Attribution License, which permits unrestricted use, distribution, and reproduction in any medium, provided the original work is properly cited.

The photocatalytic activity of substrate-supported $\mathrm{ZnO}$ nanowires is investigated with a focus on the substrate effect and the photodegradation kinetics. The employed NWs are grown on various substrates by thermal evaporation and a $50 \mu \mathrm{M}$ rhodamine $\mathrm{B}$ solution is used as the target solution. It is found that the photocatalytic activity is facilitated by using a conductive substrate and such a result can be reasonably attributed to the improvement of charge separation. The photodegradation is found to follow a zeroth-order kinetics at high concentrations and a first-order kinetics at low concentrations with a transition at around $10 \mu \mathrm{M}$. The present observation is opposed to the commonly accepted first-order kinetics. Degradation constants of $0.58 \mu \mathrm{M} \cdot \mathrm{min}^{-1}$ for the zeroth-order and $0.028 \mathrm{~min}^{-1}$ for the first-order have been achieved. The first-order constant also compares favorably with reported values.

\section{Introduction}

Photodegradation of organic pollutants based on photocatalytic nanomaterials has been receiving growing research interests in recent years due to its application for environmental protection and sustainable development [1-3]. $\mathrm{TiO}_{2}$ and $\mathrm{ZnO}$ nanomaterials are most popular and numerous photodegradation results with the use of the two nanomaterials in the suspension form have been reported in the literature [4-8]. The main drawback of the use of nanomaterials in the suspension form is that they need to be separated afterwards for cleanup and/or reuse. On the other hand, substrate-supported nanomaterials can overcome this drawback but at the cost of worse performance due to less surface area. To increase the surface area, it is natural to employ substrate-grown one-dimensional nanostructures. Photocatalytic activities of substrate-supported nanotubes, nanorods, and nanowires (NWs) of $\mathrm{TiO}_{2}$ and $\mathrm{ZnO}$ have also been investigated extensively [9-17].

It is known that the photocatalytic efficiency can be enhanced by improving charge separation between the photogenerated charge carriers [3, 15-17]. In principle, charge separation can be facilitated by using a conductive substrate for substrate-supported nanomaterials. However, experimental works on the substrate effect have been absent in the literature to our knowledge. Another concern is the photodegradation kinetics, which is widely accepted as a firstorder kinetics [5-7, 10-12]. Nevertheless, the possibility of a combined zeroth- and first-order kinetics has been proposed in a previous report [15], but no follow-up works have been reported.

The present work aims to provide more experimental results to investigate the photocatalytic activity of $\mathrm{ZnO} N W s$ with a focus on the substrate effect and the photodegradation kinetics. The employed NWs are grown on conductive and nonconductive substrates by thermal evaporation without seed layers or catalysts. The photocatalytic activity is evaluated by degrading a $50 \mu \mathrm{M}$ rhodamine $\mathrm{B}(\mathrm{RhB})$ solution $[10,11,17]$ under the irradiation of a $254 \mathrm{~nm}$ light source. The results reveal that the photocatalytic activity can be enhanced by using a conductive substrate. Also, the photodegradation follows a zeroth-order kinetics at high concentrations and a first-order kinetics at low concentrations with a transition at around $10 \mu \mathrm{M}$. Degradation constants of $0.58 \mu \mathrm{M} \cdot \mathrm{min}^{-1}$ (zeroth-order) and $0.028 \mathrm{~min}^{-1}$ (first-order) have been achieved. 


\section{Experimental}

2.1. $\mathrm{ZnO} \mathrm{NW}$ Growth. The $\mathrm{ZnO} \mathrm{NWs}$ were grown in a standard double-tube furnace system by high-temperature thermal evaporation based on a surface-roughness-assisted vapor-solid mechanism without the employment of seed layers or catalysts [18-20]. The substrates included sapphire (c-plane), Si (with doping), and fluorine-doped tin oxide (FTO), and their resistivities were around $10^{14}, 10^{-2}$, and $10^{-4} \Omega \cdot \mathrm{cm}$, respectively. To increase the substrate surface roughness for the NW growth, the sapphire and the Si (native oxide removed first in a diluted HF solution) substrates were etched prior to the growth process in $1 \mathrm{M} \mathrm{NaOH}$ and $1 \mathrm{M}$ $\mathrm{KOH}$ solutions for $1 \mathrm{~min}$, respectively. Details of the growth parameters can be found in our previous reports [18-20]. For the FTO and the Si substrates, two batches were prepared and the NW samples are designated as FTO-A, FTO-B, Si$\mathrm{A}$, and $\mathrm{Si}-\mathrm{B}$. The morphology of the NWs was examined by a scanning electron microscope (SEM; JSM 6500F, JEOL) and their photoluminescence (PL) properties were measured by a spectrometer (F-7000, Hitachi) with a $325 \mathrm{~nm}$ light source.

2.2. Photodegradation Measurement. The NW photocatalytic activity was evaluated by degrading a $50 \mu \mathrm{M}$ RhB solution. In each experimental round, a $1.5 \times 1.5 \mathrm{~cm}^{2} \mathrm{NW}$ sample was placed in a beaker with $7.5 \mathrm{~mL}$ RhB. Three $4 \mathrm{~W}$ lowpressure $\mathrm{Hg}$ lamps at a wavelength of $254 \mathrm{~nm}$ were utilized as the irradiation source, and the light intensity on the sample was $3.3 \mathrm{~mW} \cdot \mathrm{cm}^{-2}$. A small motor was employed to stir the solution and a $50 \mu \mathrm{L}$ drop was taken every 30 min during photodegradation. The absorption spectra of the drops were measured with the use of an optical microscope (BX51, Olympus), equipped with a fiber-connected spectrometer (BRC111A, BWTek).

\section{Result and Discussion}

3.1. $\mathrm{ZnO} N \mathrm{NW}$ Morphology. Figure 1 shows SEM images of $\mathrm{ZnO} \mathrm{NWs}$ grown on FTO (Figure 1(a) for FTO-A and Figure 1(c) for FTO-B), sapphire (Figure 1(d)), and $\mathrm{Si}$ (Figure 1(e) for Si-A and Figure 1(f) for Si-B). Figure 1(b) is a cross-sectional SEM image of the FTO-A sample and it displays that the NWs are straight and grow out of the substrate, which also applies to other samples. The insets in Figure 1 are enlarged images of individual NWs and it can be seen that the NW diameters range between 30 and $90 \mathrm{~nm}$. In addition, all the NWs have a good hexagonal shape and are, therefore, single-crystalline. The single-crystallinity has been well characterized in our previous reports [18-20] and will not be elaborated here.

As the photocatalysis occurs on the nanomaterial surface, the surface area of the nanomaterial is important. A convenient parameter for evaluating this property is called the roughness factor (RF), which is defined as the ratio of the surface area of the nanomaterial to the surface area of the substrate. The NWs in FTO-A have an average diameter of around $80 \mathrm{~nm}$ (i.e., a hexagonal edge width of $40 \mathrm{~nm}$ ) judging from the inset in Figure 1(a) and a length of around $6 \mu \mathrm{m}$
TABLE 1: A comparison of diameters, number densities, and RFs of the NW samples. The NW length is around $6 \mu \mathrm{m}$ for all samples.

\begin{tabular}{lccc}
\hline Sample & Diameter $(\mathrm{nm})$ & Density $\left(\mu \mathrm{m}^{-2}\right)$ & RF \\
\hline FTO-A & $70 \sim 90$ & 3.4 & 5 \\
FTO-B & $30 \sim 50$ & 7.0 & 5 \\
Sapphire & $30 \sim 50$ & 7.7 & 5.5 \\
Si-A & $50 \sim 70$ & 4.1 & 4.5 \\
Si-B & $50 \sim 70$ & 3.3 & 4 \\
\hline
\end{tabular}

from Figure 1(b). The number density is roughly $3.4 \mu \mathrm{m}^{-2}$ by carefully counting the number of NWs in an enlarged SEM image, and the RF of FTO-A is consequently estimated to be 5 . (The RF obtained by the present calculation is a rough estimate and the uncertainty is supposedly around 30\%.) A comparison of diameters, area densities, and RFs for all samples is provided in Table 1 . (Note that the NW length is around $6 \mu \mathrm{m}$ for all samples.) It can be seen that the surface areas of the NW samples are in a small range.

3.2. Substrate Effect. The absorption spectra of a $50 \mu \mathrm{M}$ RhB solution at intervals of $30 \mathrm{~min}$ due to the photocatalytic activity of the FTO-A sample are shown in Figure 2(a). The absorption peak appears at $554 \mathrm{~nm}$ and its intensity decreases down to around $5 \%$ of its original value for only $90 \mathrm{~min}$. With the use of other samples, similar absorption spectra were obtained and the peak intensities were determined. The absorption peak intensity correlates with the RhB concentration, and the ratios of the concentration as a function of time $C_{t}$ to the initial concentration $C_{0}$ for all samples as well as the results for using no NWs are plotted in Figure 2(b). As can be seen, UV irradiation alone without the assistance of NWs is ineffective to degrade RhB. On the other hand, the NWs facilitate photodegradation effectively. The FTO-A and the sapphire samples have the best and the worst performances, respectively.

There are several factors that influence the photodegradation performance including the surface area, the structural defect $[15,16]$, and the substrate conductivity. From the SEM images in Figure 1, the NWs on all substrates possess a hexagonal shape and are similarly distributed. In addition, the surface areas of the NW samples are in a close range with the sapphire sample having the highest value as can be seen in Table 1. It is convincible that the surface area is not the origin for the variation in the observed photodegradation performance.

For the role of structural defect, it is known that structural defects in $\mathrm{ZnO}$ NWs correlate with the green emission in a PL spectrum $[9,15,16,21]$. A lower PL intensity indicates a lower recombination rate of photoinduced electron-hole pairs. A lower level of structural defects is thus advantageous for photocatalysis [15]. The PL spectra are shown in Figure 3 with the peak UV intensities normalized to be equal. By comparing the PL spectra with the photodegradation curves in Figure 2(b), the two results are consistent except for the sapphire sample, which has better crystallinity than FTO$\mathrm{B}$ and $\mathrm{Si}-\mathrm{B}$ but worse performance. It is quite apparent that the worst performance of the sapphire sample arises 


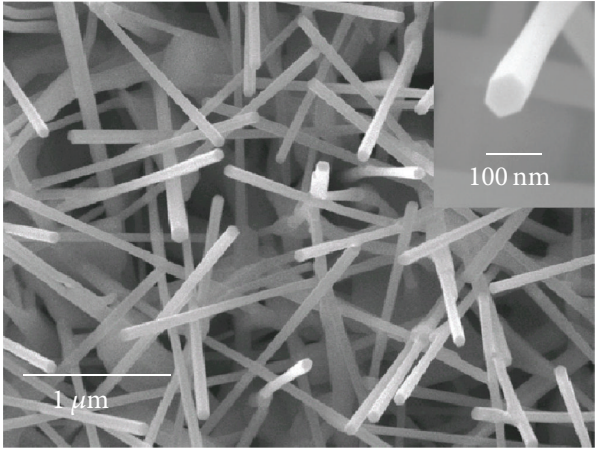

(a)

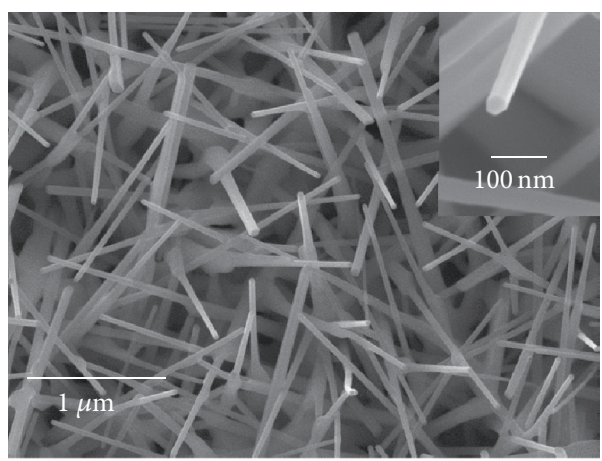

(c)

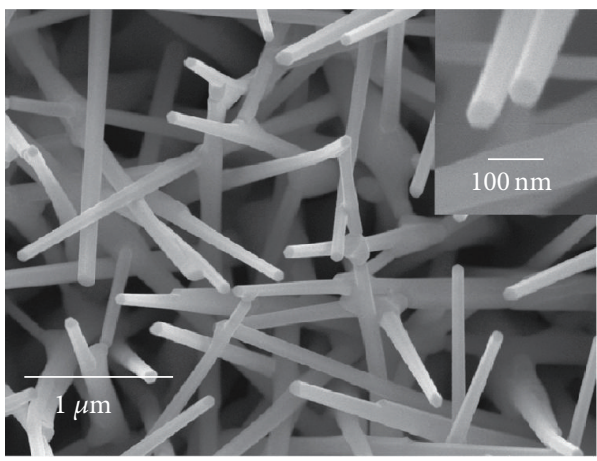

(e)

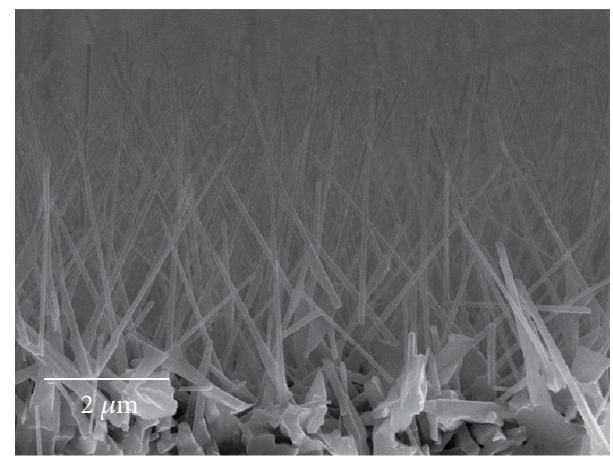

(b)

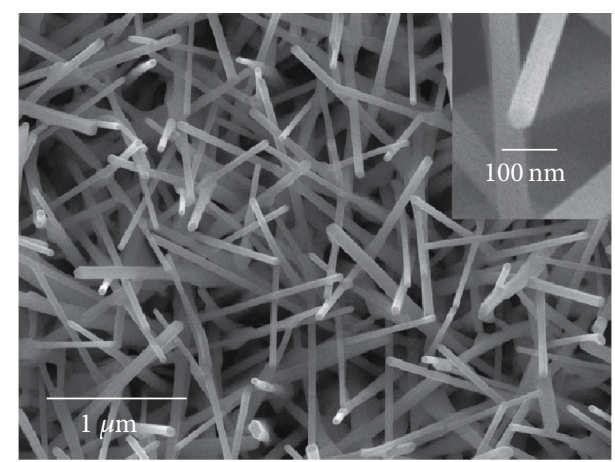

(d)

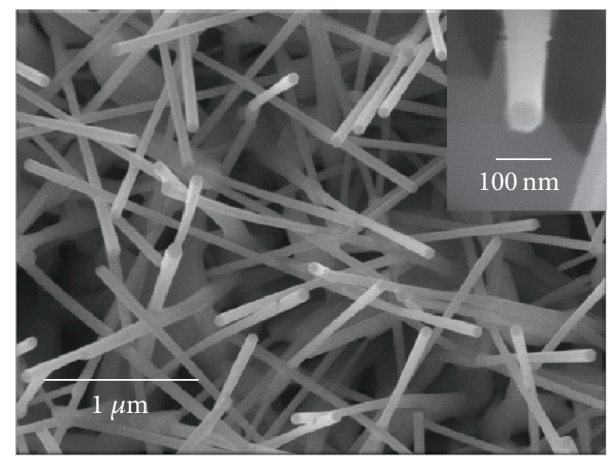

(f)

FIGURE 1: Top-view SEM images of ZnO NWs grown on ((a) and (c)) FTO (samples FTO-A and FTO-B, resp.), (d) sapphire, and ((e) and (f)) $\mathrm{Si}$ (samples Si-A and Si-B, resp.). (b) Cross-sectional SEM image of the FTO-A sample in (a). The insets reveal that the NW diameters range between 30 and $90 \mathrm{~nm}$.

from sapphire's nonconductive characteristic. Consequently, a conductive substrate is beneficial for photodegradation and such a result can be reasonably attributed to better charge separation.

3.3. Photodegradation Kinetics. The ratios of $C_{t}$ to $C_{0}$ in Figure 2(b) allow the determination of the photodegradation kinetics. It is commonly accepted that the photodegradation follows a first-order kinetics $[1,5-7,10-12,17]$ and $C_{t}$ can be expressed as

$$
C_{t}=C_{0} \exp \left(-k_{1} t\right),
$$

where $k_{1}$ is the first-order constant. To verify if this is the case, the logarithmic plots of the ratios in Figure 2(b) have been obtained. It is found that the logarithmic plots for the sapphire, Si-B, and FTO-B samples follow the linear relationship but the plots for the $\mathrm{Si}-\mathrm{A}$ and FTO-A samples deviate (see Figure 4(a)). Therefore, the first-order kinetics does not seem to be suitable.

It has been proposed and explained previously that the degradation reaction is concentration dependent, and the photodegradation follows a zeroth-order kinetics at high concentrations and a first-order kinetics at low concentrations [15]. For the zeroth-order kinetics, $C_{t}$ can be expressed as

$$
C_{t}=C_{0}-k_{0} t
$$

where $k_{0}$ is the zeroth-order constant. The ratio of $C_{t}$ to $C_{0}$ is therefore a straight line. As can be seen in Figure 2(b), 


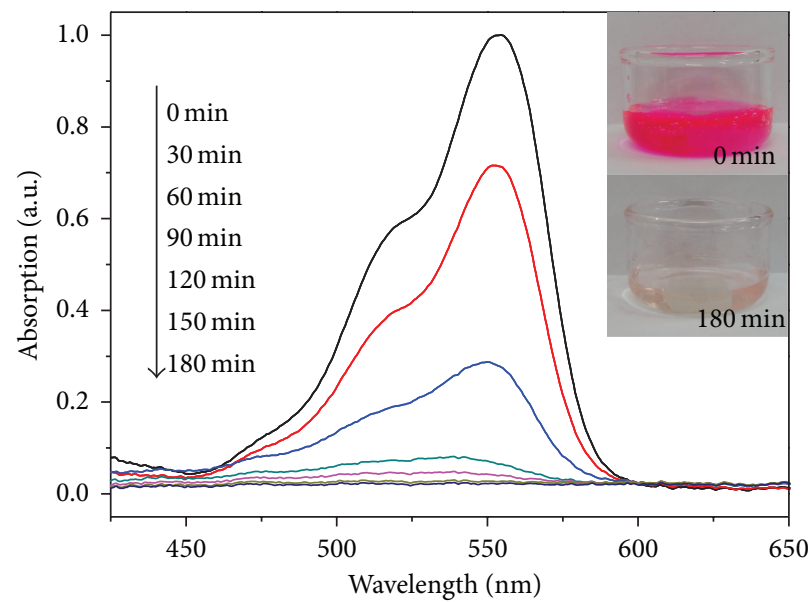

(a)

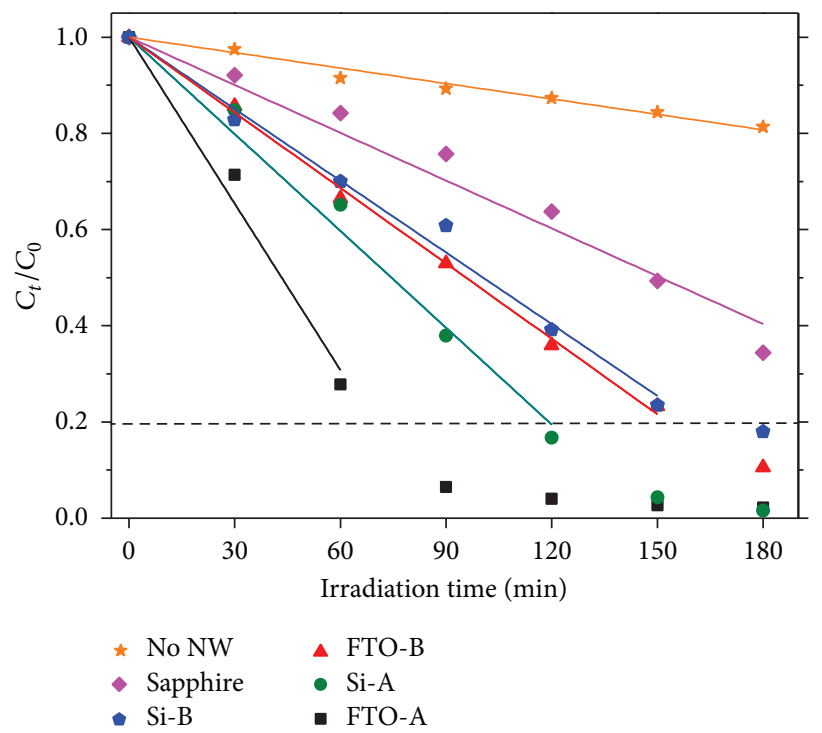

(b)

Figure 2: (a) Absorption spectra of a $50 \mu \mathrm{M}$ RhB solution at intervals of 30 min due to the photocatalytic activity of the FTO-A sample. (b) Ratios of $C_{t}$ to $C_{0}$ as a function of irradiation time for all tested NW samples and using no NWs. A transition occurs at approximately the ratio of 0.2 , which corresponds to $10 \mu \mathrm{M}$, and is indicated by a dashed line.

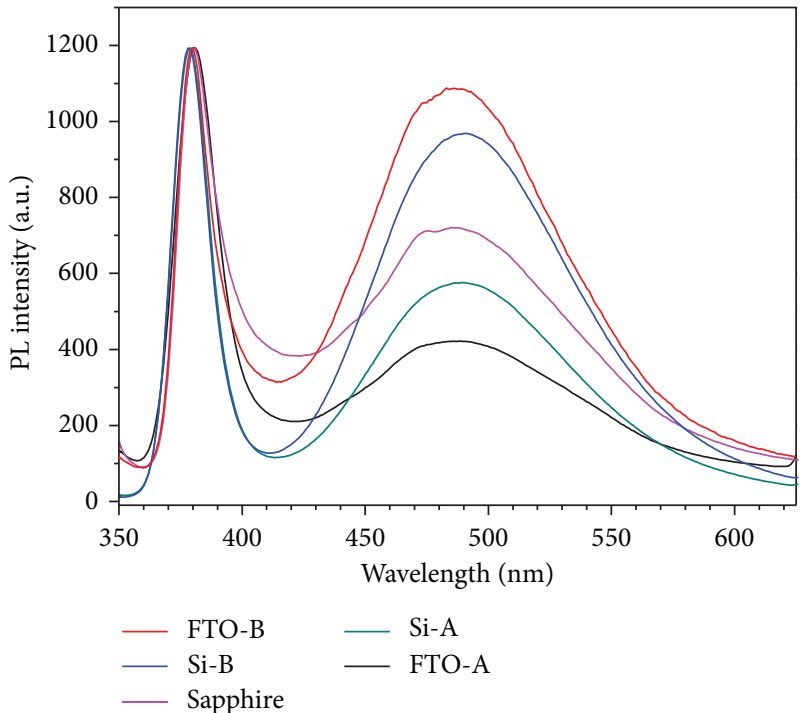

FIGURE 3: PL spectra of the NW samples with the peak UV intensities normalized to be equal.

the ratios are indeed straight lines at high concentrations but deviate at low concentrations for the Si-A and the FTO-A samples.

The logarithmic plots of the Si-A and the FTO-A ratios are shown in Figure 4(a) and they roughly have a linear relationship at low concentrations. For further confirmation, the FTO-A sample was used for another test to degrade a $10 \mu \mathrm{M}$ RhB solution. The logarithmic plot of the concentration ratios is shown in Figure 4(b), and the good linear relationship ensures the validity of the first-order kinetics at concentrations below $10 \mu \mathrm{M}$. Therefore, the present results support the combined kinetics instead of the commonly accepted first-order kinetics. Judging from the Si-A and the FTO-A ratios in Figure 2(b), the transition occurs at approximately $10 \mu \mathrm{M}$ (i.e., at the ratio of 0.2 ) and is indicated by a dashed line. Incidentally, this transition concentration of $10 \mu \mathrm{M}$ has been the initial concentration in several $\mathrm{RhB}$ photodegradation reports $[10,11,17]$. It is reasonable that the zeroth-order kinetics has not been observed in these works.

Although the theoretical basis for the combined kinetics has been described in [15], it is instructive to have a simple explanation. Since the photodegradation occurs on the NW surface, it depends on the molecular surface coverage on the NW. The surface coverage is a result of molecular adsorption and desorption and can be described by the Langmuir equation [15, 22]. At high concentrations, desorption is negligible in comparison with adsorption $[15,22]$. The NW surface is thus fully covered with molecules and the (full) coverage is independent of the concentration, which is shown in Figure 5(a). The photodegradation consequently proceeds at a constant rate regardless of the concentration and follows the zeroth-order kinetics in (2). At low concentrations, adsorption and desorption are comparable. The NW surface is thus partially covered with molecules and the coverage depends on the concentration, which is shown in Figure 5(b). The photodegradation consequently proceeds at a rate proportional to the concentration and follows the first-order kinetics in (1). The transition concentration is influenced by adsorption, desorption, and other factors as well.

The calculated zeroth-order constants from the fitted lines in Figure 2(b) are 0.054 (no NW), 0.17 (sapphire), 0.25 (SiB), 0.26 (FTO-B), 0.34 (Si-A), and 0.58 (FTO-A) $\mu \mathrm{M} \cdot \mathrm{min}^{-1}$. Since no zeroth-order constants for $\mathrm{RhB}$ photodegradation 


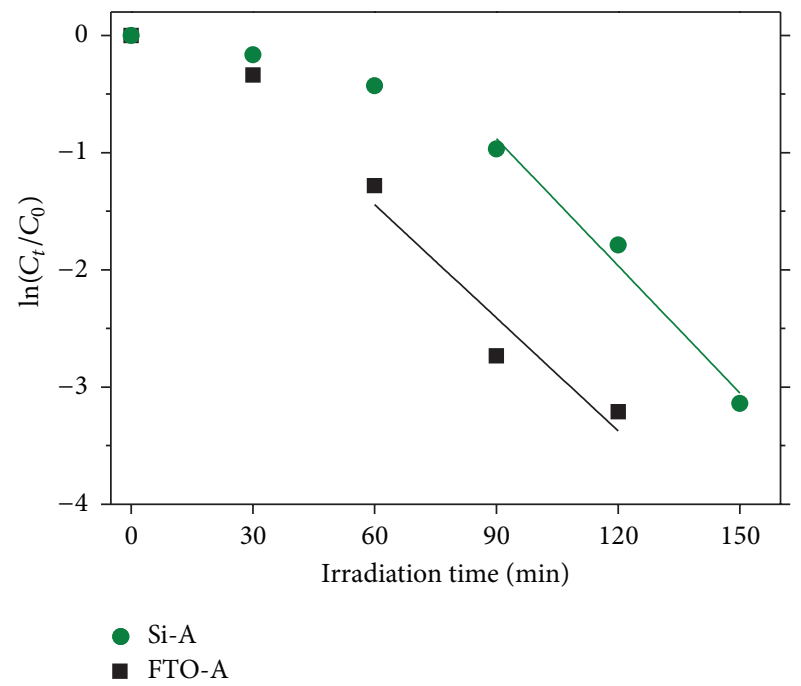

(a)

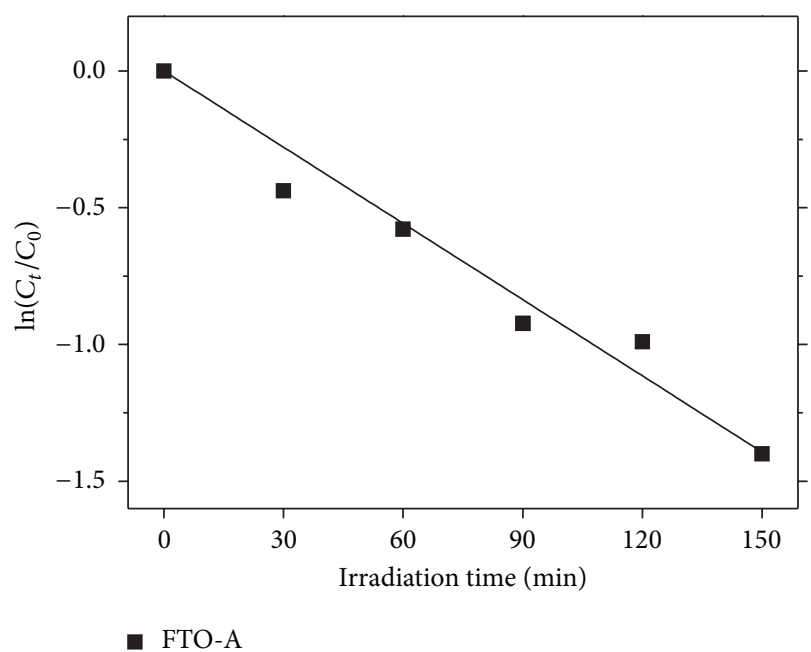

(b)

FIGURE 4: Logarithmic plots of concentration ratios of (a) a $50 \mu \mathrm{M}$ solution due to the photocatalysis of the Si-A and the FTO-A samples as shown in Figure 2(b) and (b) a $10 \mu \mathrm{M}$ solution due to the FTO-A sample.

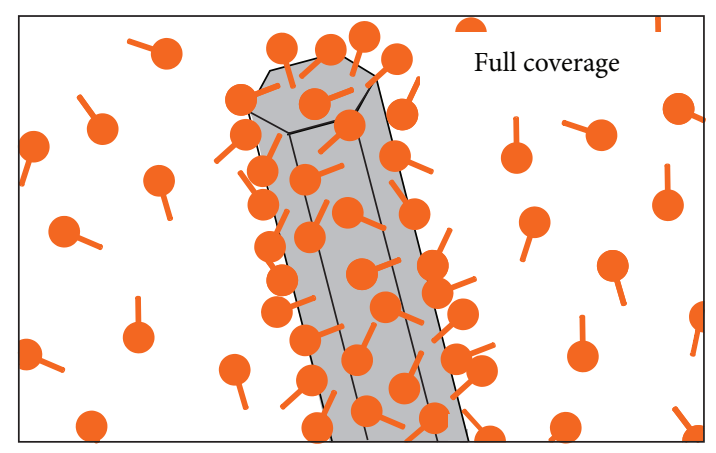

(a)

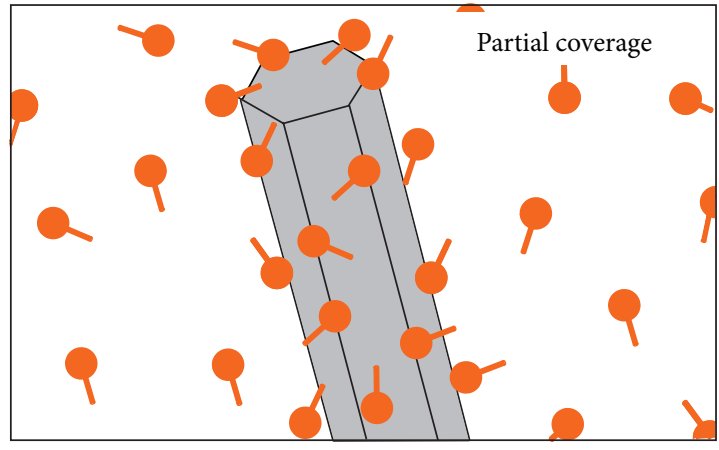

(b)

FIGURE 5: (a) The NW surface is fully covered with molecules at high concentrations and the (full) coverage is independent of the concentration. (b) The NW surface is partially covered with molecules at low concentrations and the coverage depends on the concentration.

are available in the literature, these constants cannot be compared. On the other hand, the first-order constants are roughly 0.036 (Si-A) and $0.032 \mathrm{~min}^{-1}$ (FTO-A) from the fitted lines in Figure 4(a). A more accurate result from the fitted line in Figure 4(b) is $0.028 \mathrm{~min}^{-1}$ for the FTO-A sample. It is noteworthy that this first-order constant of $0.028 \mathrm{~min}^{-1}$ is higher than reported values of around $0.01 \mathrm{~min}^{-1}$ at a similar ratio of solution volume to NW substrate area of around $3 \mathrm{~mL} \cdot \mathrm{cm}^{-2}[10,11,17]$.

\section{Conclusions}

The present work investigates the photocatalytic activity of $\mathrm{ZnO}$ NWs with a focus on the substrate effect and the photodegradation kinetics. The employed NWs are grown on nonconductive and conductive substrates by thermal evaporation. The photocatalytic activity is evaluated by degrading a $50 \mu \mathrm{M}$ RhB solution under the irradiation of a $254 \mathrm{~nm}$ light source. The results reveal that the photocatalytic activity can be enhanced by using a conductive substrate due to improved charge separation. The degradation kinetics has been found to follow a zeroth-order kinetics at high concentrations and a first-order one at low concentrations with a transition at around $10 \mu \mathrm{M}$. Degradation constants of $0.58 \mu \mathrm{M} \cdot \mathrm{min}^{-1}$ for the zeroth-order and $0.028 \mathrm{~min}^{-1}$ for the first-order have been achieved. The first-order constant also compares favorably with reported values of around $0.01 \mathrm{~min}^{-1}$ in the literature.

\section{Conflict of Interests}

The authors declare that there is no conflict of interests regarding the publication of this paper.

\section{Acknowledgment}

This work was supported by the National Science Council under Grant no. 101-2112-M-007-016. 


\section{References}

[1] M. R. Hoffmann, S. T. Martin, W. Y. Choi, and D. W. Bahnemann, "Environmental applications of semiconductor photocatalysis," Chemical Reviews, vol. 95, no. 1, pp. 69-96, 1995.

[2] U. I. Gaya and A. H. Abdullah, "Heterogeneous photocatalytic degradation of organic contaminants over titanium dioxide: a review of fundamentals, progress and problems," Journal of Photochemistry and Photobiology C: Photochemistry Reviews, vol. 9, no. 1, pp. 1-12, 2008.

[3] C. C. Chen, W. H. Ma, and J. C. Zhao, "Semiconductormediated photodegradation of pollutants under visible-light irradiation," Chemical Society Reviews, vol. 39, no. 11, pp. 42064219, 2010.

[4] S. Sakthivel, B. Neppolian, M. V. Shankar, B. Arabindoo, M. Palanichamy, and V. Murugesan, "Solar photocatalytic degradation of azo dye: Comparison of photocatalytic efficiency of $\mathrm{ZnO}$ and $\mathrm{TiO}_{2}$," Solar Energy Materials and Solar Cells, vol. 77, no. 1, pp. 65-82, 2003.

[5] M. A. Behnajady, N. Modirshahla, and R. Hamzavi, "Kinetic study on photocatalytic degradation of C.I. Acid Yellow 23 by ZnO photocatalyst," Journal of Hazardous Materials, vol. 133, no. 1-3, pp. 226-232, 2006.

[6] D. Mijin, M. Savić, P. Snežana et al., "A study of the photocatalytic degradation of metamitron in $\mathrm{ZnO}$ water suspensions," Desalination, vol. 249, no. 1, pp. 286-292, 2009.

[7] Y. A. Chung, Y. C. Chang, M. Y. Lu, C. Y. Wang, and L. J. Chen, "Synthesis and photocatalytic activity of small-diameter $\mathrm{ZnO}$ nanorods," Journal of the Electrochemical Society, vol. 156, no. 5, pp. F75-F79, 2009.

[8] B. X. Li and Y. F. Wang, "Facile synthesis and enhanced photocatalytic performance of flower-like $\mathrm{ZnO}$ hierarchical microstructures," Journal of Physical Chemistry C, vol. 114, no. 2, pp. 890-896, 2010.

[9] M. Y. Guo, M. K. Fung, F. Fang et al., " $\mathrm{ZnO}$ and $\mathrm{TiO}_{2}$ 1D nanostructures for photocatalytic applications," Journal of Alloys and Compounds, vol. 509, no. 4, pp. 1328-1332, 2011.

[10] T. J. Kuo, C. N. Lin, C. L. Kuo, and M. H. Huang, "Growth of ultralong $\mathrm{ZnO}$ nanowires on silicon substrates by vapor transport and their use as recyclable photocatalysts," Chemistry of Materials, vol. 19, no. 21, pp. 5143-5147, 2007.

[11] G. Wang, D. Chen, H. Zhang, J. Z. Zhang, and J. H. Li, “Tunable photocurrent spectrum in well-oriented zinc oxide nanorod arrays with enhanced photocatalytic activity," Journal of Physical Chemistry C, vol. 112, no. 24, pp. 8850-8855, 2008.

[12] X. M. Zhao, B. H. Zhang, K. L. Ai et al., "Monitoring catalytic degradation of dye molecules on silver-coated $\mathrm{ZnO}$ nanowire arrays by surface-enhanced Raman spectroscopy," Journal of Materials Chemistry, vol. 19, no. 31, pp. 5547-5553, 2009.

[13] C. L. Wu, L. Shen, H. G. Yu, Y. C. Zhang, and Q. L. Huang, "Solvothermal synthesis of $\mathrm{Cu}$-doped $\mathrm{ZnO}$ nanowires with visible light-driven photocatalytic activity," Materials Letters, vol. 74, pp. 236-238, 2012.

[14] Y. X. Wang, X. Y. Li, G. Lu, X. Quan, and G. H. Chen, "Highly oriented 1-D ZnO nanorod arrays on zinc foil: direct growth from substrate, optical properties and photocatalytic activities," The Journal of Physical Chemistry C, vol. 112, no. 19, pp. 73327336, 2008.

[15] Y. Liu, Z. H. Kang, Z. H. Chen et al., "Synthesis, characterization, and photocatalytic application of different $\mathrm{ZnO}$ nanostructures in array configurations," Crystal Growth and Design, vol. 9, no. 7, pp. 3222-3227, 2009.
[16] D. W. Chu, Y. Masuda, T. Ohji, and K. Kato, "Formation and photocatalytic application of $\mathrm{ZnO}$ nanotubes using aqueous solution," Langmuir, vol. 26, no. 4, pp. 2811-2815, 2010.

[17] J. Bae, J. B. Han, X. M. Zhang et al., "ZnO nanotubes grown at low temperature using ga as catalysts and their enhanced photocatalytic activities," Journal of Physical Chemistry C, vol. 113, no. 24, pp. 10379-10383, 2009.

[18] S. T. Ho, K. C. Chen, H. A. Chen, H. Y. Lin, C. Y. Cheng, and H. N. Lin, "Catalyst-free surface-roughness-assisted growth of large-scale vertically aligned zinc oxide nanowires by thermal evaporation," Chemistry of Materials, vol. 19, no. 16, pp. 40834086, 2007.

[19] S. T. Ho, C. Y. Wang, H. L. Liu, and H. N. Lin, "Catalystfree selective-area growth of vertically aligned zinc oxide nanowires," Chemical Physics Letters, vol. 463, no. 1-3, pp. 141$144,2008$.

[20] S. T. Ho, C. L. Hsiao, H. Y. Lin, H. A. Chen, C. Y. Wang, and H. N. Lin, "Single-crystalline zinc oxide nanowires as photoanode material for dye-sensitized solar cells," Journal of Nanoscience and Nanotechnology, vol. 10, no. 10, pp. 6473-6478, 2010.

[21] M. Kashif, U. Hashim, M. E. Ali et al., "Effect of different seed solutions on the morphology and electrooptical properties of ZnO Nanorods," Journal of Nanomaterials, vol. 2012, Article ID 452407, 6 pages, 2012.

[22] R. Desikan, S. Armel, H. M. Meyer III, and T. Thundat, "Effect of chain length on nanomechanics of alkanethiol self-assembly," Nanotechnology, vol. 18, no. 42, Article ID 424028, 2007. 

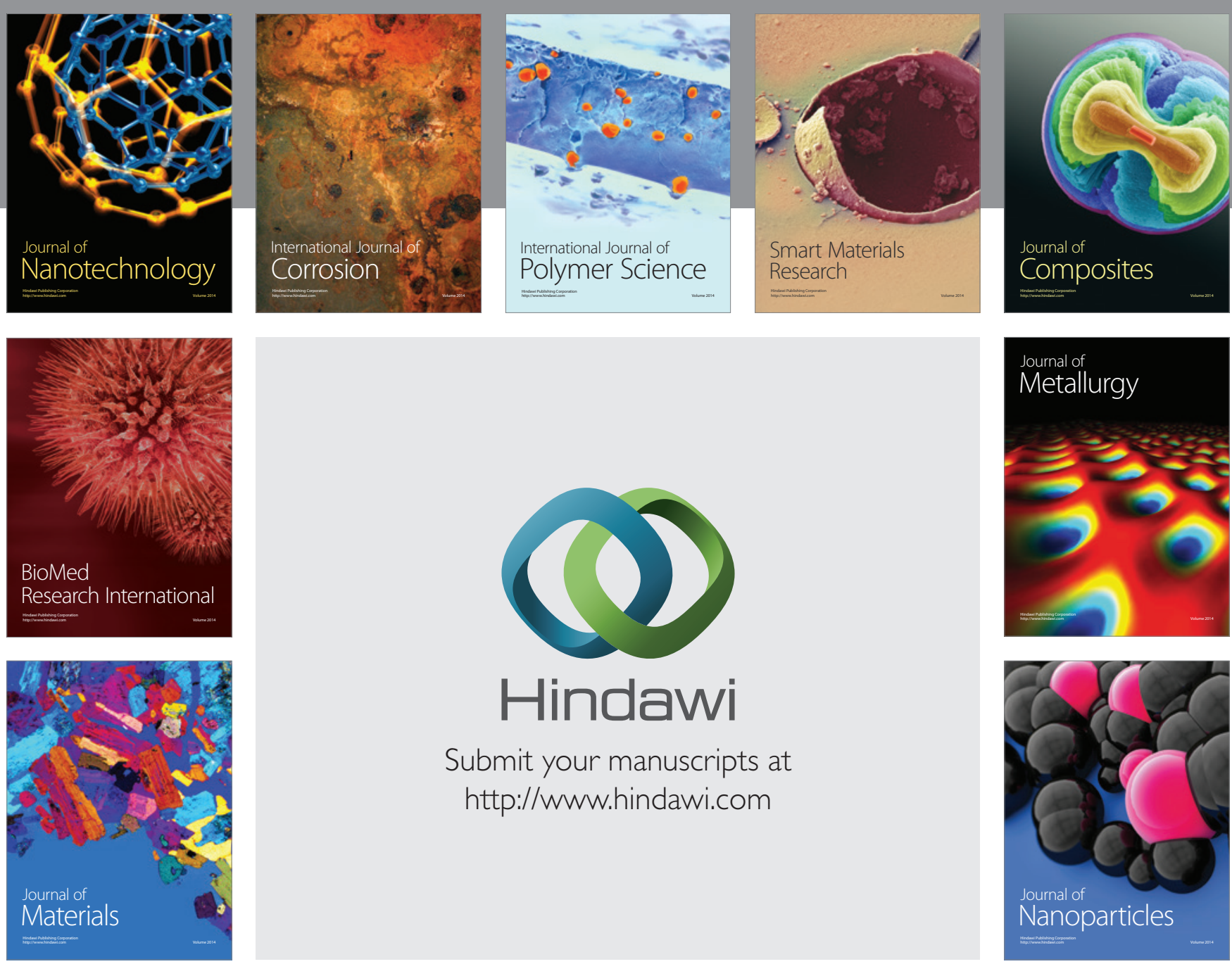

Submit your manuscripts at http://www.hindawi.com
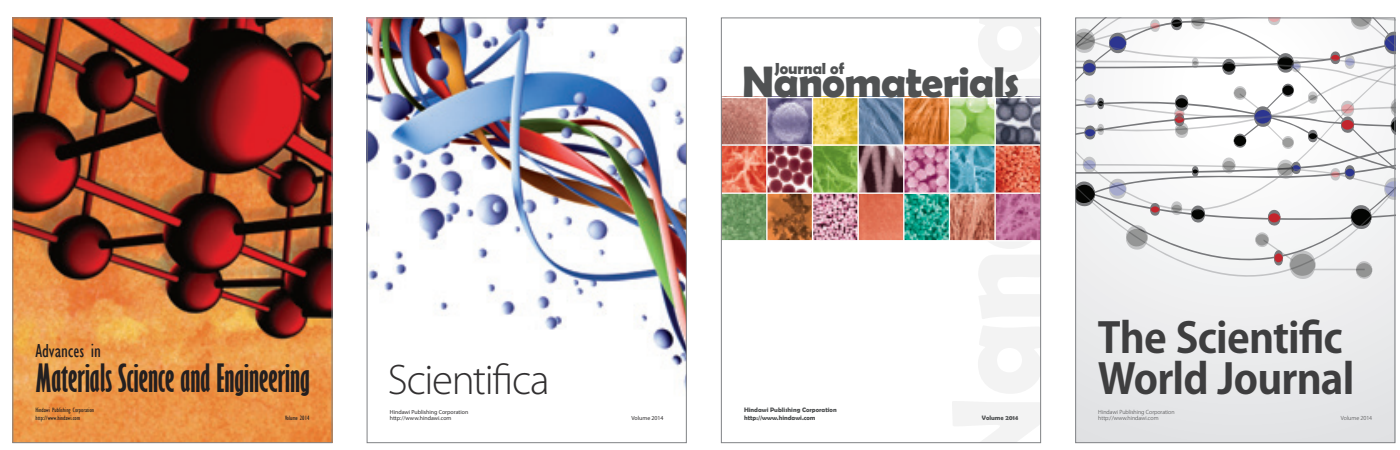

\section{The Scientific World Journal}
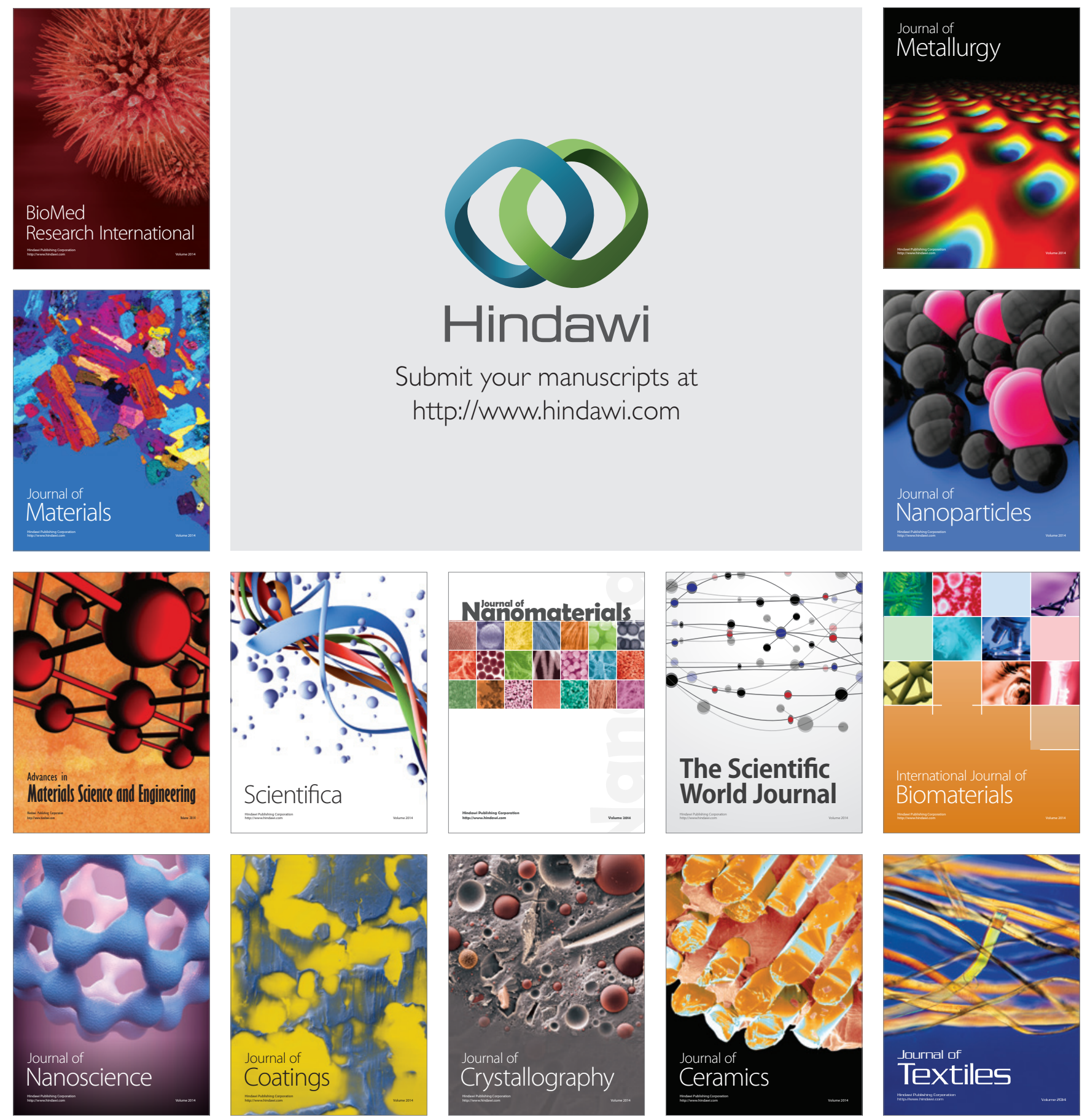